\title{
The millimeter and submillimeter wave spectrum of cis-methyl vinyl ether
}

\author{
A.M. Daly ${ }^{1}$, L. Kolesniková, S. Mata, J.L. Alonso ${ }^{\Uparrow}$ \\ Grupo de Espectroscopía Molecular (GEM), Edificio Quifima, Laboratorios de Espectroscopia y Bioespectroscopia, Parque Científico UVa, Unidad Asociada CSIC, Universidad \\ de Valladolid, 47011 Valladolid, Spain
}

\begin{abstract}
Among the species of potential interstellar relevance, methyl vinyl ether $\left(\mathrm{CH}_{3} \mathrm{OCH} @ \mathrm{CH}_{2}\right)$ is the simplest ether compound containing both alkyl and alkene functional groups. In order to facilitate its detection in the ISM, we have measured the millimeter and submillimeter wave spectra from 50 to $650 \mathrm{GHz}$. We present the analysis of pure rotational spectrum of the cis-methyl vinyl ether in the vibrational ground state and in the first excited states of in-plane bending mode $\left(\mathbf{m}_{16}\right)$ and methyl $\left(\mathbf{m}_{23}\right)$ and skeletal $\left(\mathbf{m}_{24}\right)$ torsional modes. Coriolis and Fermi type interactions between the $V_{24}=1$ and $V_{23}=1$ states have been explicitly treated using an effective two-state Hamiltonian.
\end{abstract}

\section{Introduction}

Grain-surface and gas-phase reactions have been found as the main reaction mechanisms of the chemical formation of complex organic molecules in hot regions of star formation [1-3]. According to the gas-phase models, the granular ices rich in methanol, ethanol, and higher alcohols, are evaporated into the gas phase and undergo alkyl cation transfer reactions followed by dissociative recombination reactions leading to the formation of neutral oxygen containing organic species like ethers $[1,4]$. Astronomical detections of dimethyl ether [5], methyl ethyl ether [6], tentative detection of diethyl ether [7] as well as the observation of vinyl alcohol [8] in the star-forming regions, place methyl vinyl ether, the simplest ether compound containing both alkyl and alkene functional groups, among the species of potential interstellar relevance. Possible detection of the methyl vinyl ether in the ISM would be greatly helped by its previous laboratory measurements and spectral analysis.

Previously published laboratory works on the rotational spectroscopy of the most stable cis-conformer of the methyl vinyl ether (see Fig. 1a) include the pure rotational transitions below $40 \mathrm{GHz}$ in frequency [9-13]. In the first microwave study by Cahill et al.

\footnotetext{
$\Uparrow$ Corresponding author. Fax: +34 983186349 .

E-mail address: jlalonso@qf.uva.es (J.L. Alonso).

Present address: Jet Propulsion Laboratory, California Institute of Technology, 4800 Oak Grove Dr., Pasadena, CA 91109, United States.
}

[9], a set of the ground state rotational constants for the cis-methyl vinyl ether was reported. Later on, Kaushik [10], Damiani et al. [11], Fujitake and Hayashi [12], and Meyer et al. [13] extended the assignments up to $\mathrm{J}^{00}=66$ and $\mathrm{K}_{\mathrm{a}}^{00}=31$ and provided a centrifugal distortion analysis for the vibrational ground state. In addition, the substitution structure was derived on the basis of the rotational constants obtained for 14 isotopologues [12] and electric dipole moment components $\left|\mathbf{l}_{\mathrm{a}}\right|=0.295$ (1) $\mathrm{D}$ and $\left|\mathbf{l}_{\mathrm{b}}\right|=0.910$ (2) D were determined from Stark measurements $[9,12]$. The small A-E splittings arising from the coupling between methyl internal rotation and the overall rotation were resolved in the vibrational ground state using waveguide FTMW spectroscopy and a barrier to internal rotation of methyl group of $1256 \mathrm{~cm}^{-1}$ was derived [13]. Cis-methyl vinyl ether has three low-lying vibrational modes $[14,15]$ involving skeletal CCAOC torsion $\left(\mathrm{m}_{24}\right), \mathrm{OACH}_{3}$ torsion $\left(\mathrm{m}_{23}\right)$ and in-plane CAOAC bending mode $\left(\mathrm{m}_{16}\right)$, depicted in Fig. 1a. Gas phase IR and Raman studies of this molecule give the corresponding fundamentals at $234,244.5$ and $327 \mathrm{~cm}^{-1}$, respectively [14]. Assignments and analysis of the rotational transitions in some excited vibrational states have been also reported [9,11-13]. The pure rotational spectrum of the less stable gauche-conformer was published by McWhorter et al. [16].

A plausible interstellar identification of methyl vinyl ether should be based on directly measured transitions or transitions predicted from a fit including higher frequency data. In this paper we report measurements and analysis of more than 4500 lines of the cis-methyl vinyl ether up to $650 \mathrm{GHz}$. Precise sets of spectroscopic 


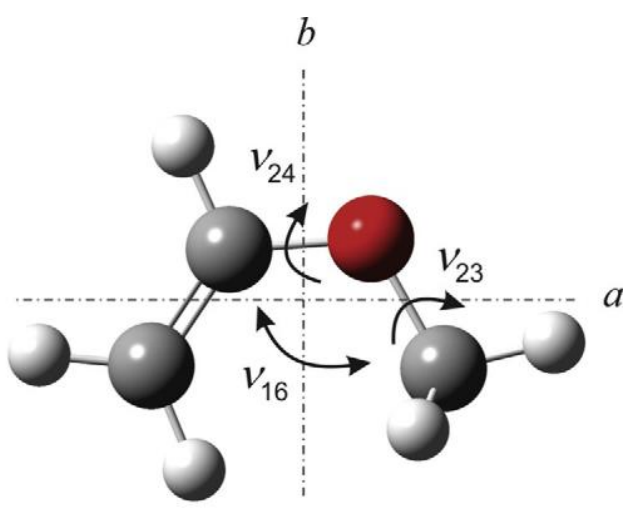

(a)

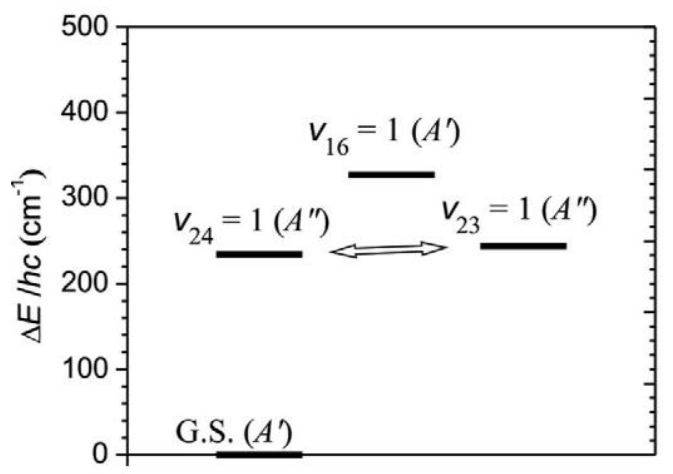

(b)

Fig. 1. (a) Cis-methyl vinyl ether molecule depicted in the ab inertial plane with schematic illustration of the three low-lying vibrational modes. (b) Energy differences between the ground and first excited states of the three low-lying vibrational modes of the cis-methyl vinyl ether obtained from IR experiments [14]. The arrow indicates a mutual interaction between $V_{24}=1$ and $V_{23}=1$ states observed and analyzed in this work.

constants for the vibrational ground state, first excited state of the bending mode $\left(\mathbf{V}_{16}=1\right)$ as well as those resulting from the coupled fit between the first excited states of methyl and skeletal torsions $\left(V_{23}=1\right.$ and $V_{24}=1$, respectively) have been determined. Results of this work should provide sufficiently precise laboratory information to enable astronomers to search for the cis-methyl vinyl ether towards various interstellar sources over a wide range of frequencies. The experimental setup, recently constructed at the University of Valladolid, used in the present study as well as in the previous works on ethyl cyanide [17], vinyl cyanide [18], phenol [19], or ethyl mercaptan [20], is described in the next section.

\section{Experimental details}

Schemes of the two configurations of the millimeter and submillimeter wave spectrometer used in this work covering the spectral range from 50 to $1080 \mathrm{GHz}$ are shown in Fig. 2. The microwave source (1) is a frequency modulated signal generator (Agilent E8257D) operating from $250 \mathrm{kHz}$ to $20 \mathrm{GHz}$, phase locked to a Rubidium standard (2). In the double pass configuration (Fig. 2a), the microwave frequency is multiplied by 4,6 or 9 using amplifier multiplier chains AMC WR15, AMC WR10, or AMC WR6.5 (3) from VDI, Inc., with average powers of $34 \mathrm{~mW}, 17 \mathrm{~mW}$, and $4 \mathrm{~mW}$, to reach the frequency ranges of $50-75 \mathrm{GHz}, 75-110 \mathrm{GHz}$ or 110 $170 \mathrm{GHz}$, respectively. The optical path length of the free space cell (4) of $3.6 \mathrm{~m}$ is doubled using a polarization grid (5) (Millitech, Inc.) and a home-made rooftop mirror (6). The output signal, after the second pass through the cell, is detected by the corresponding solid state Schottky diodes detectors WR15, WR10 and WR6.5 (7) from VDI, Inc. Rotational spectra above $170 \mathrm{GHz}$ are measured in a single pass mode configuration shown in Fig. $2 \mathrm{~b}$ using parabolic metal mirrors (Edmund Optics, Inc.) as focusing optic elements (8). An active VDI sextupler AMC WR9.0 (average power of $25 \mathrm{~mW}$ ) is initially used to produce a suitable frequency input for additional active and passive multipliers (VDI, Inc.) connected by waveguide to its output (9). The active doublers WR4.3 and WR 2.2 are connected to a $12 \mathrm{~V}$ bias implemented directly in the WR9.0 while the passive triplers, WR 2.8, WR 1.5 , and WR1.0 require no bias. Using combinations of these multipliers, the frequencies of 170 $240 \mathrm{GHz}$ (WR 4.3), 240-360 GHz (WR2.8), 340-480 GHz (WR4.3 and WR2.2), $510-720 \mathrm{GHz}$ (WR4.3 and WR1.5), and 720$1080 \mathrm{GHz}$ (WR2.8 and WR1.0) are reached with average powers of $3 \mathrm{~mW}, 0.9 \mathrm{~mW}, 0.2 \mathrm{~mW}, 50 \mathrm{lW}$, and $9 \mathrm{lW}$, respectively. A broadband Quasi-Optical Detector (QOD) from VDI, Inc. and liquid helium cooled silicon bolometer (Infrared Laboratories, Inc.) are used as the detection elements (10). For both configurations, detected source frequency-modulated signal is amplified (11) and send to the lock-in amplifier (SR510, Stanford Research Systems, Inc.) where $2 \mathrm{f}$ detection is applied, where $\mathrm{f}$ is the modulation frequency, to increase the sensitivity of the measurements (12). Modulation frequency of $10.2 \mathrm{kHz}$ is used for the non-cooled (room temperature) detectors while $90 \mathrm{~Hz}$ for the cryogenic detector and the modulation depth is determined to give the Doppler line width. The generator and the lock-in amplifier are connected by standard GPIB to the computer (13) and controlled by a laboratory developed LabVIEW program.

The commercially available sample of gaseous methyl vinyl ether (ChemSampCo, LLC, 98\% purity) was used without further purification. The rotational spectra were recorded at room temperature with pressure of approximately 20 lbar in the frequency region $50-650 \mathrm{GHz}$. All the spectra were recorded in $1 \mathrm{GHz}$ sections in both directions and further processed using the AABS package [21]. The transition lines were fit using a Gaussian profile function with accuracy for isolated well-developed lines estimated to be better than $50 \mathrm{kHz}$.

\section{Ground state rotational spectrum}

Cis-methyl vinyl ether is a prolate asymmetric top with symmetry properties described by $\mathrm{C}_{\mathrm{s}}$ point group. Assignments of the ground state transitions were begun by making predictions based on the lower frequency data [12,13]. Dominant features of the spectrum are series of b-type R-branch transitions separated about $2 \mathrm{C} 9.7 \mathrm{GHz}$ value with the most intense $(\mathrm{J}+1)_{1 \mathrm{~J}+1} \mathrm{~J}_{0 \mathrm{~J}}$ and $(\mathrm{J}+1)_{0 \mathrm{~J}+1} \mathbf{J}_{1 \mathrm{~J}}$ pairs which are degenerate with increasing $\mathrm{J}$ quantum number. These transitions are accompanied with recognizable spectral patterns of b-type Q-branch series whose head lines are separated by approximately $2 \mathrm{~A}-\mathrm{B}-\mathrm{C} 25 \mathrm{GHz}$ value. Much weaker a-type R-branch transitions were identified as well. Once the new data were assigned, they were added to the lower frequency transitions to form a global data set consisting of more than 2800 measured a-type and b-type transitions involving the rotational quantum numbers $\mathrm{J}^{00}$ and $\mathrm{K}_{\mathrm{a}}^{00}$ up to 69 and 25, respectively. Rotational transitions were analyzed with the asymmetric-top Hamiltonian in the Watson's A-reduction in $\mathrm{I}^{\mathrm{r}}$-representation [22] using SPFIT and SPCAT programs [23]. All the transition frequencies introduced to the least-square analysis were weighted inversely proportional to the second power of their estimated uncertainties. The unresolved pairs of the asymmetry splitting or transitions overlapped accidentally were fit as the intensity weighted averages. No A-E splittings due to the internal rotation were observed in the measured ground state transitions. Spectroscopic constants resulting from the final fit are summarized in Table 1 and the 
Millimeter- and submillimeter-wave spectrometr at the University of Valladolid

(a) Double Pass Configuration $(50-170 \mathrm{GHz})$

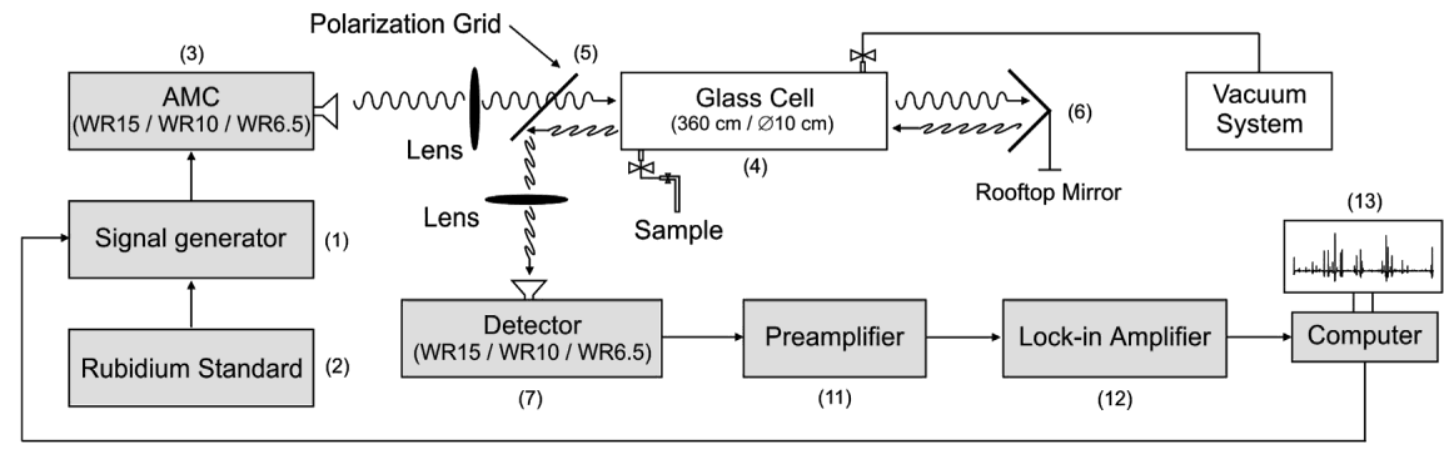

(b) Single Pass Configuration $(170-1080 \mathrm{GHz})$

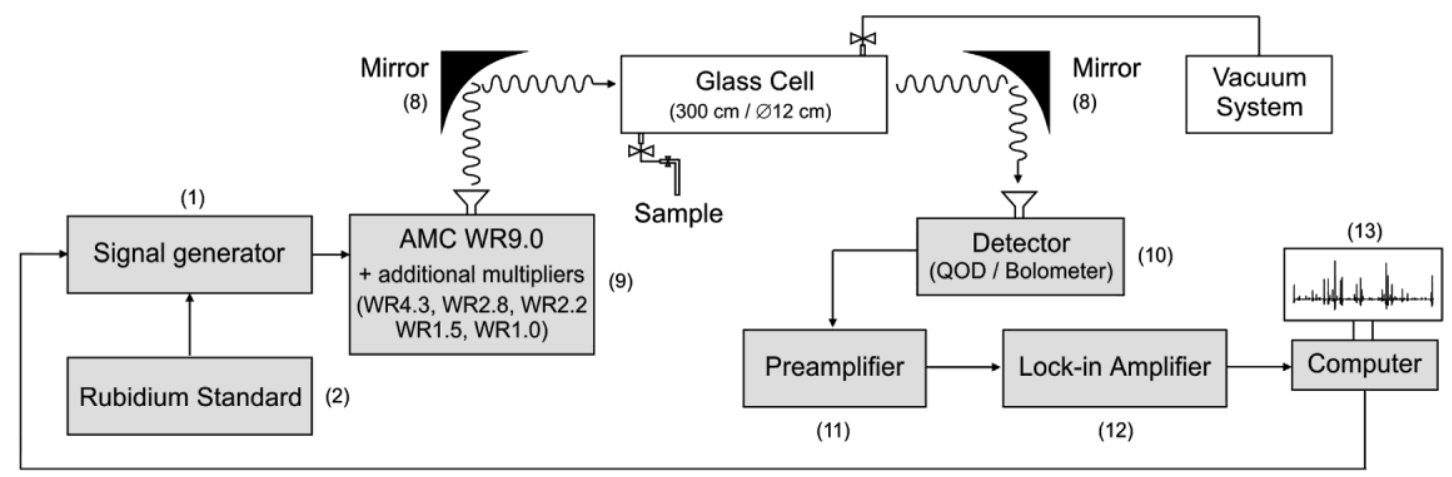

Fig. 2. Schemes of the single (a) and double path (b) configurations of the millimeter- and submillimeter wave spectrometer at the University of Valladolid.

complete list of the rotational transitions is given in Table $\mathrm{S} 1$ of the electronic supplementary material. The rms deviation of the fit of $32 \mathrm{kHz}$ is comparable to the experimental accuracy. The present dataset allows a determination of a complete set of the sextic centrifugal distortion constants and significantly refines the rotational and quartic centrifugal distortion constants given in the original microwave studies (see Table 1). These newly derived spectroscopic constants provide an accurate prediction of the cis-methyl vinyl ether in the millimeter and submillimeter wave region that can be used to guide future observational searches.

\section{Rotational spectra in the excited states}

\subsection{Vibrational satellites}

The first excited states of the three lowest-energy modes, $\mathbf{m}_{24}$, $\mathrm{m}_{23}$, and $\mathrm{m}_{16}$, were sufficiently populated at the room temperature of the experiment to readily observe rotational satellite lines. A section of the rotational spectrum showing the satellite pattern of the $16_{116} \quad 15_{0} \quad 15$ and $16_{016} \quad 15_{1} \quad 15$ pair is presented in Fig. 3. Assignments of the rotational transitions in the excited states were carried out under the support of the Loomis-Wood type plots which were used successfully as a tool for the excited states assignments in other systems $[19,21,24]$. An example of this plot is shown in Fig. 4 where all transitions are lined up to the central frequencies of the ground state $(\mathrm{J}+1)_{1 \mathrm{~J}+1} \quad \mathbf{J}_{0 \mathrm{~J}}$ transitions and each $\mathrm{K}_{\mathrm{a}}{ }^{0} \quad \mathrm{~K}_{\mathrm{a}}{ }^{00}$ sequence then forms a visible stripe running from lower to upper values of J quantum numbers (see Fig. 4). Stripes assigned to the $\mathrm{K}_{\mathrm{a}}^{0} \quad \mathrm{~K}_{\mathrm{a}}^{00}=1 \quad 0$ sequence for the ground state as well as for $\mathbf{V}_{24}=1, \mathbf{V}_{23}=1$, and $\mathbf{V}_{16}=1$ states are highlighted. For the $\mathbf{V}_{23}=1$ state, this sequence shows a discontinuity between $\mathrm{J}^{00}=17$ and 21 (see Fig. 4) which might be attributed to an interaction with other neighboring state. Fig. $1 \mathrm{~b}$ gives evidence of small energy separation between $V_{23}=1$ and $V_{24}=1$ of $10.5 \mathrm{~cm}^{-1}$ [14] which may allow considerable perturbations in the pure rotational spectra of both states. Their analysis is given in the following section.

The $\mathrm{V}_{16}=1$ excited state appears to be free of interactions according to its energy spacing with respect to $\mathrm{V}_{23}=1$ and the $\mathbf{V}_{24}=1$ states. Spectral assignments of the transitions in the $V_{16}=1$ state to quantum numbers were started with the lowest $\mathrm{K}_{\mathrm{a}}$ transitions identified using the Loomis-Wood type plots. As new data were assigned, they were continuously added to the previous data set [12] and fit using Watson's A-reduced semirigid Hamiltonian [22] in $I^{\mathrm{r}}$-representation, initially up to the fourth order. As the analysis proceeded, sextic centrifugal distortion terms were tested and retained if they were well determined and produced any significant improvement in the fit. Only $U_{\mathrm{IK}}, \mathrm{U}_{\mathrm{KJ}}$, $U_{K}, I$, and $Y$ could be determined with reasonable accuracy. An appreciable number of $\mathrm{R}$ - and Q-branch series of transitions in the $V_{16}=1$ state were observed split by internal rotation (magnitude in units of $\mathrm{MHz}$ ), which is in agreement with the splitting observed in Ref. [13], however these transitions were not included into the analysis. The spectroscopic constants resulting from the fit of more than 500 a-type and b-type rotational transitions $\left(\mathrm{J}^{00}\right.$ and $\mathrm{K}_{\mathrm{a}}{ }^{00}$ up to 59 and 15 , respectively) are summarized in Table 1 . The complete list of the rotational transitions is given in Table S2 of the electronic supplementary material.

\subsection{Coupled analysis of the $V_{24}=1$ and $V_{23}=1$ states}

The lowest $\mathrm{K}_{\mathrm{a}}$ sequences of the b-type R-branch transitions in the $\mathbf{V}_{23}=1$ and $\mathbf{V}_{24}=1$ states, identified in Fig. 4, together with 
Table 1

Spectroscopic constants of the cis-methyl vinyl ether (A-reduction, $\mathrm{I}^{\mathrm{r}}$-representation) ${ }^{\mathrm{a}}$ for the vibrational ground state and $\mathbf{V}_{16}=1, \mathbf{V}_{23}=1$ and $\mathbf{V}_{24}=1$ excited states

\begin{tabular}{|c|c|c|c|c|c|c|c|c|c|c|}
\hline \multirow[t]{2}{*}{ Constant } & \multirow[t]{2}{*}{ Unit } & \multirow{2}{*}{$\begin{array}{l}\text { This work } \\
\text { Ground state }\end{array}$} & \multirow[b]{2}{*}{$V_{16}=1$} & \multirow[b]{2}{*}{$\mathrm{V}_{23}=1$} & \multirow[b]{2}{*}{$V_{24}=1$} & \multirow{2}{*}{$\begin{array}{l}\text { Damiani et. al. [11] } \\
\text { Ground state }\end{array}$} & \multirow[b]{2}{*}{$\mathrm{V}_{16}=1$} & \multirow[b]{2}{*}{$\mathrm{V}_{23}=1$} & \multirow[b]{2}{*}{$\mathbf{V}_{24}=1$} & \multirow{2}{*}{$\begin{array}{l}\text { Meyer et. al. [13] } \\
\text { Ground state }\end{array}$} \\
\hline & & & & & & & & & & \\
\hline A & $(\mathrm{MHz})$ & $18224.9674(1)$ & $18395.7066(9)$ & $18301.884(3)$ & $18223.0386(5)$ & $18224.981(6)$ & $18395.73(1)$ & $18301.54(2)$ & 18222.79 (2) & $18224.99(1)$ \\
\hline B & $(\mathrm{MHz})$ & 6388.99209 (3) & $6365.8442(2)$ & 6330.9979 (5) & $6359.0810(1)$ & $6388.992(2)$ & $6365.86(1)$ & $6331.298(7)$ & $6359.43(2)$ & $6389.002(4)$ \\
\hline $\mathrm{C}$ & $(\mathrm{MHz})$ & 4875.85998 & $4854.33587(9)$ & $4855.6062(1)$ & $4875.15309(9)$ & $4875.8621(6)$ & 4854.355 (2) & $4861.871(2)$ & 4868.877 (3) & 4875.867 (3) \\
\hline$D_{\jmath}$ & $(\mathrm{kHz})$ & $3.85336(2)$ & $3.8669(1)$ & $3.7435(4)$ & $3.8693(1)$ & $3.84(7)$ & $4.4(3)$ & $3.8(2)$ & $4.2(3)$ & $3.861(9)$ \\
\hline $\mathrm{D}_{\mathrm{JK}}$ & $(\mathrm{kHz})$ & $-11.5212(1)$ & $-13.760(2)$ & $-10.215(8)$ & $-11.495(2)$ & $-10.4(5)$ & $-13.9(4)$ & $-35.8(5)$ & $7.5(6)$ & $-11.41(6)$ \\
\hline$D_{K}$ & $(\mathrm{kHz})$ & $49.5624(3)$ & $56.44(1)$ & $50.11(6)$ & $49.210(7)$ & 47 (4) & $58(2)$ & $68(6)$ & $39(5)$ & $49.46(2)$ \\
\hline $\mathrm{d}_{\mathrm{J}}$ & $(\mathrm{kHz})$ & $1.183668(7)$ & 1.20168 (7) & $1.1383(2)$ & $1.18190(6)$ & $1.23(2)$ & $1.19(2)$ & $1.24(2)$ & $1.18(3)$ & $1.1842(9)$ \\
\hline$d_{K}$ & $(\mathrm{kHz})$ & $6.2388(2)$ & $8.540(2)$ & $4.49(1)$ & $5.233(3)$ & $4.6(5)$ & $8.6(6)$ & $-5.6(4)$ & $20.7(5)$ & $6.30(5)$ \\
\hline $\mathrm{U}_{\mathrm{J}}$ & $(\mathrm{mHz})$ & $-1.138(4)$ & $\ldots$ & $-1.138^{\mathrm{b}}$ & $-1.138^{\mathrm{b}}$ & $\ldots$ & $\ldots$ & $\ldots$ & $\ldots$ & $\ldots$ \\
\hline $\mathrm{U}_{\mathrm{JK}}$ & $(\mathrm{mHz})$ & $71.9(1)$ & $160(1)$ & $71.9^{\mathrm{b}}$ & $71.9^{\mathrm{b}}$ & $\ldots$ & $\ldots$ & $\ldots$ & $\ldots$ & $\ldots$ \\
\hline $\mathrm{U}_{\mathrm{KJ}}$ & $(\mathrm{mHz})$ & $-450.0(4)$ & $-790(14)$ & $-450.0^{\mathrm{b}}$ & $-450.0^{\mathrm{b}}$ & $\ldots$ & $\ldots$ & $\ldots$ & $\ldots$ & $\ldots$ \\
\hline $\mathrm{U}_{\mathrm{K}}$ & $(\mathrm{mHz})$ & $883.5(5)$ & $1300(31)$ & $883.5^{\mathrm{b}}$ & $883.5^{\mathrm{b}}$ & $\ldots$ & $\ldots$ & $\ldots$ & $\ldots$ & $290(30)$ \\
\hline$/ \mathrm{J}$ & $(\mathrm{mHz})$ & $-0.243(1)$ & $0.208(8)$ & $-0.243^{\mathrm{b}}$ & $-0.243^{\mathrm{b}}$ & $\ldots$ & $\ldots$ & $\ldots$ & & $\ldots$ \\
\hline$/{ }_{\mathrm{JK}}$ & $(\mathrm{mHz})$ & $10.58(8)$ & $\ldots$ & $10.58^{\mathrm{b}}$ & $10.58^{\mathrm{b}}$ & $\ldots$ & $\ldots$ & $\ldots$ & $\ldots$ & $\ldots$ \\
\hline /к & $(\mathrm{mHz})$ & $254(1)$ & $1321(34)$ & $254^{\mathrm{b}}$ & $254^{\mathrm{b}}$ & $\ldots$ & $\ldots$ & $\ldots$ & $\ldots$ & $-580(70)$ \\
\hline \multirow[t]{2}{*}{$\mathrm{DE}$} & $(\mathrm{MHz})$ & $\ldots$ & & $305924.3(1)$ & $\ldots$ & $\ldots$ & $\ldots$ & $\ldots$ & $\ldots$ & \\
\hline & $\left(\mathrm{cm}^{-1}\right)$ & $\ldots$ & & $10.204536(3)$ & $\ldots$ & $\ldots$ & $\ldots$ & $\ldots$ & $\ldots$ & \\
\hline $\mathrm{G}_{\mathrm{c}}$ & (MHz) & $\ldots$ & & $1381^{\mathrm{c}}$ & $\ldots$ & $\ldots$ & $\ldots$ & $\ldots$ & $\ldots$ & \\
\hline$G_{c}^{J}$ & (MHz) & $\ldots$ & & 0.00661 & $\ldots$ & $\ldots$ & $\ldots$ & $\ldots$ & $\ldots$ & \\
\hline $\mathrm{G}_{\mathrm{c}}^{\mathrm{K}}$ & (MHz) & $\ldots$ & & $-0.503(1)$ & & $\ldots$ & $\ldots$ & $\ldots$ & & \\
\hline$G_{c}^{K K}$ & $(\mathrm{MHz})$ & $\ldots$ & & $0.000062(2)$ & $\ldots$ & $\ldots$ & $\ldots$ & $\ldots$ & $\ldots$ & \\
\hline $\mathrm{F}_{\mathrm{ab}}$ & $(\mathrm{MHz})$ & $\ldots$ & & $-7.204(3)$ & $\ldots$ & $\ldots$ & $\ldots$ & $\ldots$ & $\ldots$ & \\
\hline $\mathrm{F}^{\mathrm{K}}$ & $(\mathrm{MHz})$ & $\cdots$ & & $-0.00268(2)$ & $\cdots$ & $\cdots$ & $\cdots$ & $\cdots$ & $\ldots$ & \\
\hline $\mathrm{W}_{ \pm}$ & $(\mathrm{MHz})$ & $\ldots$ & & $1.19445(5)$ & $\ldots$ & $\ldots$ & $\ldots$ & $\ldots$ & $\ldots$ & \\
\hline $\mathrm{N}_{\text {lines }} / \mathrm{N}_{\mathrm{ex}}{ }^{\mathrm{d}}$ & & 2838/9 & $501 / 0$ & $352 / 6$ & $884 / 14$ & 41 & 29 & 27 & 26 & 55 \\
\hline $\mathrm{J}^{\mathrm{vw}}{ }_{\text {max }}$ & & 69 & 59 & 59 & 61 & 22 & 17 & 10 & 12 & 66 \\
\hline $\mathrm{K}_{\mathrm{a}}^{\mathrm{OUO}}{ }_{\max }$ & & 25 & 15 & 6 & 10 & 9 & 8 & 3 & 3 & 31 \\
\hline $\mathbf{r}_{\text {fit }}{ }^{e}$ & $(\mathrm{kHz})$ & 32 & 32 & 44 & 40 & 90 & 70 & 70 & & 78 \\
\hline
\end{tabular}

${ }^{\text {a }}$ The numbers in parentheses are $1 \mathrm{r}$ uncertainties in the units of the last decimal digit.

${ }^{\mathrm{b}}$ Fixed to the ground state values.

Fixed to the value derived from the harmonic force field (see the text).

Number of the distinct frequency lines in the fit/number of the excluded lines based on the $3 u$ and $5 u$ criterions for the ground state and excited states fits, respectively, where $u$ is the uncertainty of the line.

' Root mean square deviation of the fit. 


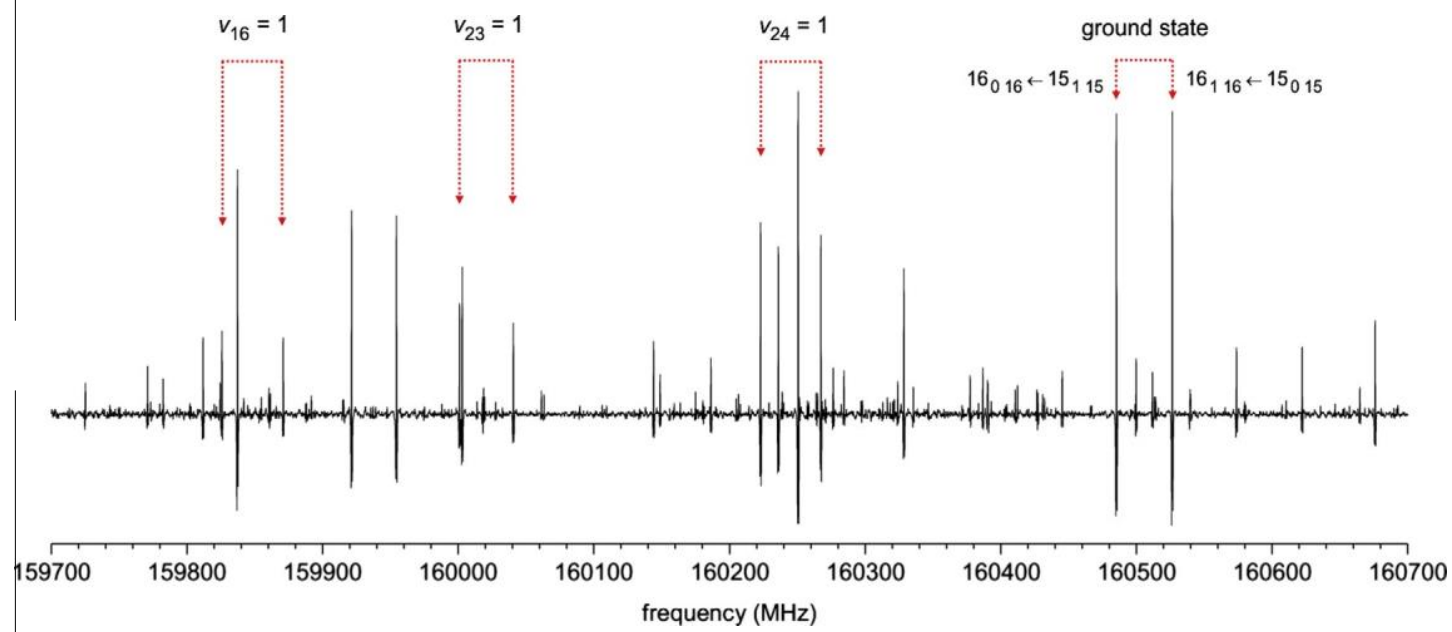

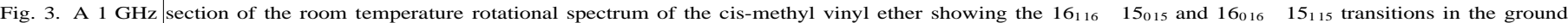

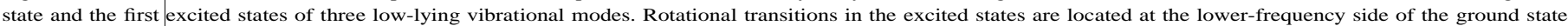
lines.

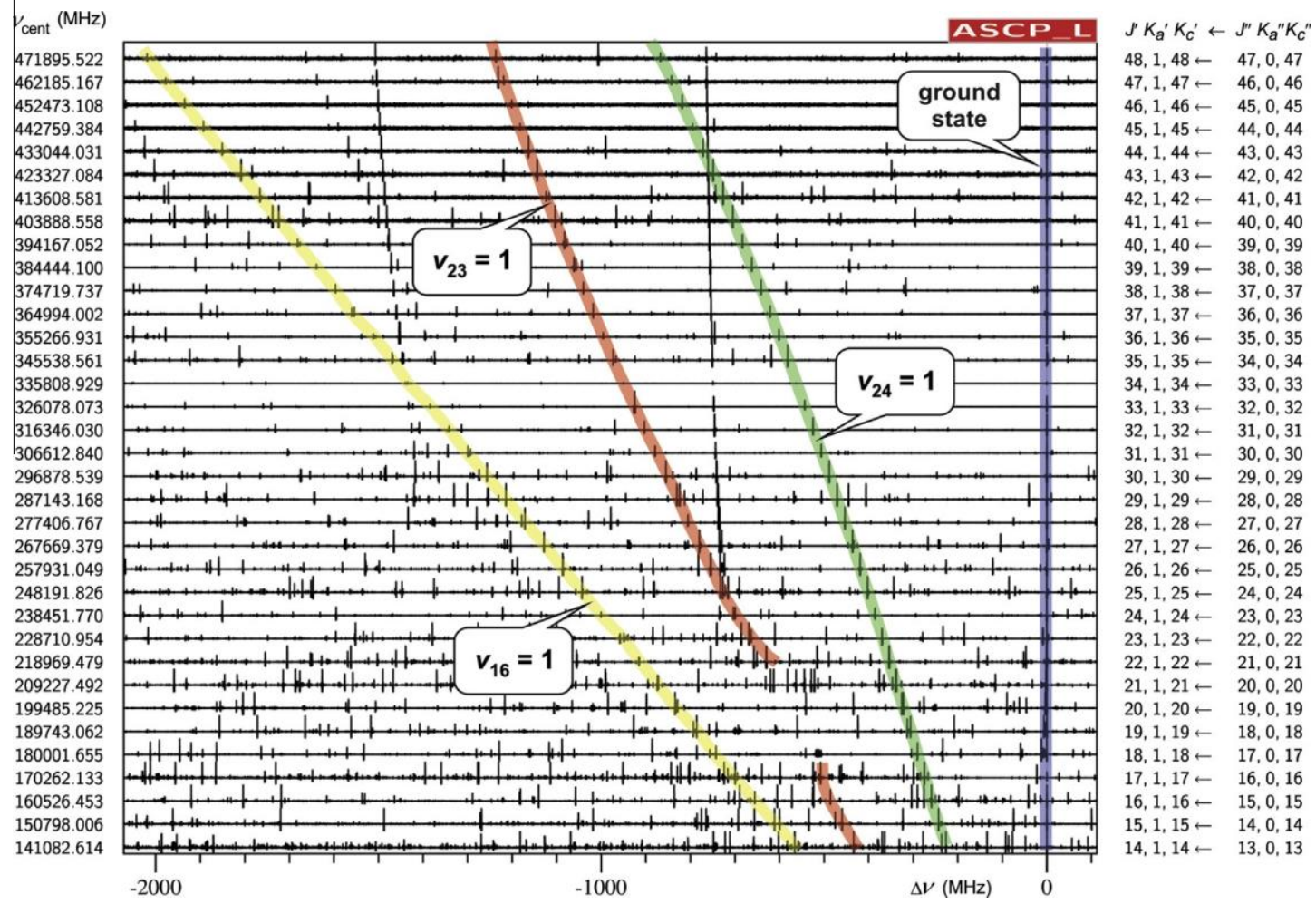

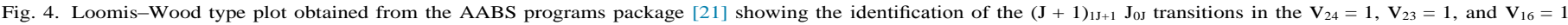

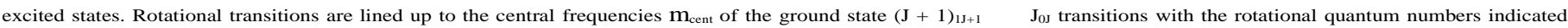
on the right side of the diagram. The frequency distance $\mathrm{Dm}$ from the central frequency $\mathrm{m}_{\mathrm{cent}}$ is documented on the $\mathrm{x}$-axis.

transitions measured previously $[9,12]$ were the starting points for the initial separate analysis of both excited states. In case of the $\mathbf{V}_{23}=1$ state, anomalous deviations (increasing as $\mathbf{J}$ rising) between the observed line positions and those calculated using the standard Watson's A-reduced semirigid Hamiltonian [22] were found rapidly in the $\mathrm{K}_{\mathrm{a}}^{0} \mathrm{~K}_{\mathrm{a}}^{00}=1 \quad 0$ sequence. Similar behavior, but with opposite sign of deviations, was found in the same range of the $\mathrm{J}$ quantum numbers in the $\mathrm{K}_{\mathrm{a}}{ }^{0} \quad \mathrm{~K}_{\mathrm{a}}{ }^{00}=2 \quad 3$ sequence pertaining to the $V_{24}=1$ state which clearly indicates that $V_{23}=1$ and
$\mathbf{V}_{24}=1$ states are mutually perturbed. The same anomalies revealed also corresponding a-type transitions. Departures of the quartic centrifugal distortion constants values and opposite signs of some of them when compared to the ground state give more evidence of neglected interactions. To achieve a satisfactory analysis of all measured transitions the next step was therefore a simultaneous analysis of the $\mathbf{V}_{24}=1$ and $V_{23}=1$ states using the effective Hamiltonian with explicitly considered vibration-rotation interactions. 

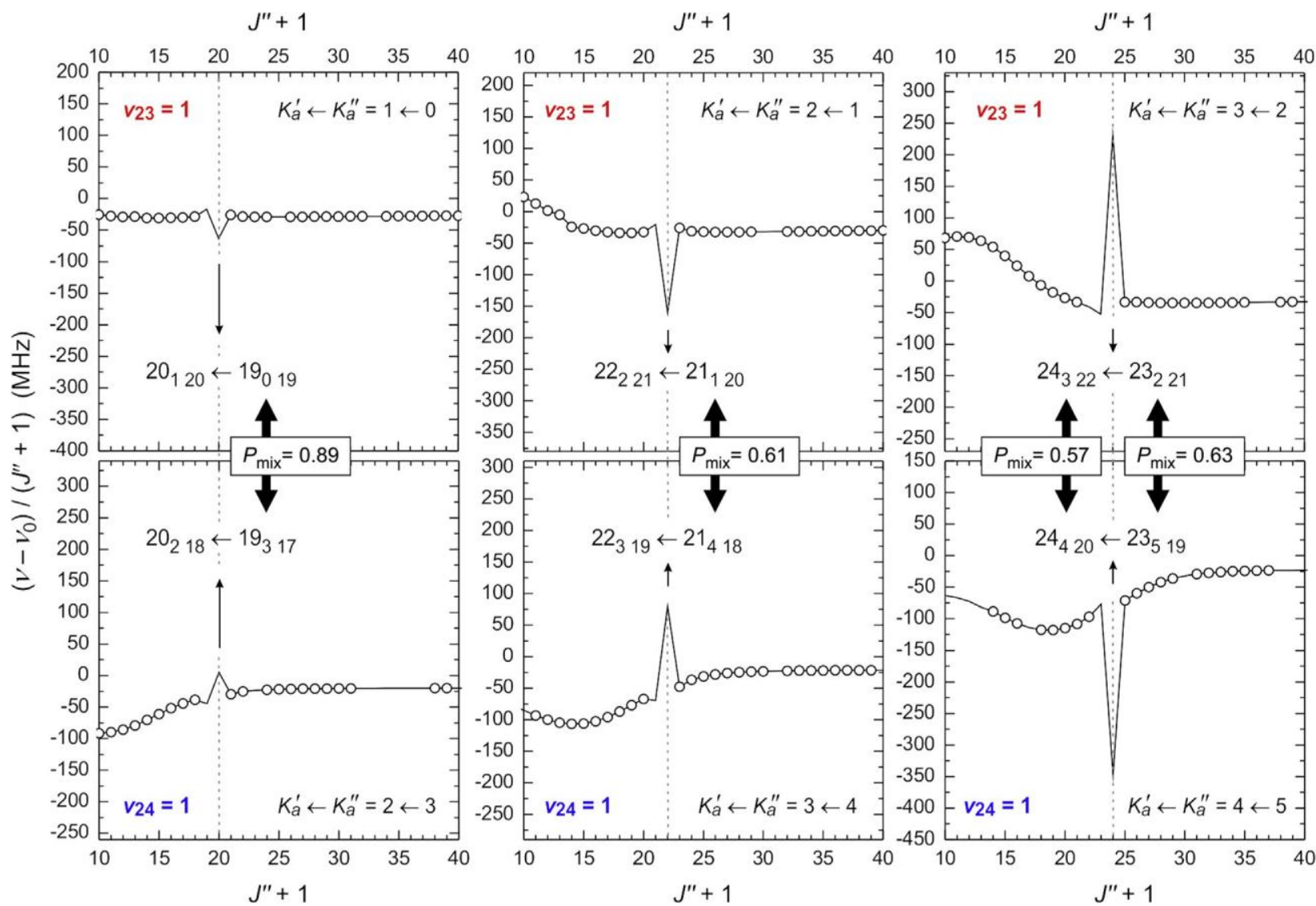

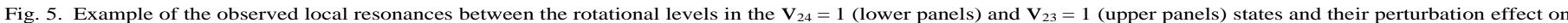

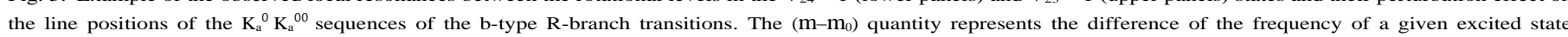

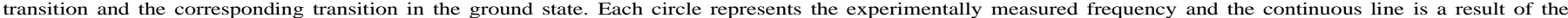

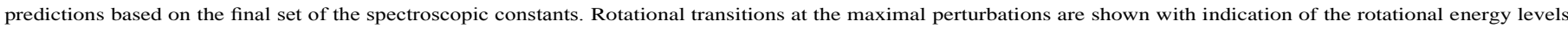
involved in the resonances. Corresponding mixing coefficients $P_{\text {mix }}$ are given for each interacting pair of the rotational energy levels.

Under the $\mathrm{C}_{\mathrm{s}}$ symmetry point group, both states belong to the same irreducible representation, $\mathrm{C}\left(\mathrm{V}_{24}=1\right)=\mathrm{C}\left(\mathrm{V}_{23}=1\right)=\mathrm{A}^{00}$, which may allow both Coriolis and anharmonic Fermi-type interactions between them. The effective two-state Hamiltonian used takes the following form

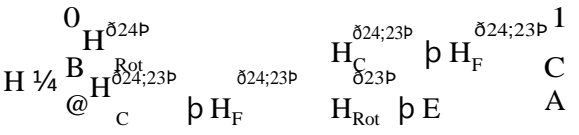

where the purely V-diagonal matrix elements are Watson's semirigid Hamiltonians $\mathrm{H}_{\mathrm{Rot}}^{(24)}$ and $\mathrm{H}_{\mathrm{Rot}}^{(23)}$ (A-reduction, $\mathrm{I}^{\mathrm{r}}$-representation) [22] for $\mathbf{V}_{24}=1$ and $\mathbf{V}_{23}=1$ states, respectively, and DE is the vibrational energy difference between both states. The off-diagonal blocs are composed from the sum of Coriolis and Fermi-type operators $\mathrm{Hc}^{(24,23)}$ and $\mathrm{HF}^{(24,23)}$, respectively. Assuming the Coriolis interaction, nonzero Coriolis terms are those for which the direct product $\mathrm{C}\left(\mathrm{V}_{24}=1\right){ }^{\circledR} \mathrm{C}\left(\mathrm{V}_{23}=1\right){ }^{\circledR} \mathrm{C}\left(\mathrm{J}_{\mathrm{a}}\right)$, where $\mathrm{a}=\mathrm{a}, \mathrm{b}, \mathrm{c}$, contains a totally symmetric representation [25]. According to this argument it can be derived that only c-type Coriolis coupling is allowed giving the Coriolis Hamiltonian up to the second power in angular momentum as follows [26]

$H^{\mathfrak{2} 24 ; 23 p} \quad 1 / 4 \mathrm{iG}_{\mathrm{c}} \mathrm{J}_{\mathrm{y}} p \mathrm{~F}_{\mathrm{ab}} \mathrm{J}_{\mathrm{z}} \mathrm{J}_{\mathrm{x}} p \mathrm{~J}_{\mathrm{x}} \mathrm{J}_{\mathrm{z}} \mathrm{p}$

where $\mathrm{G}_{\mathrm{c}}$ and $\mathrm{F}_{\mathrm{ab}}$ are the Coriolis coupling constants and the $\mathrm{z}, \mathrm{x}$, and $\mathrm{y}$ axis are taken to be the $\mathrm{a}, \mathrm{b}$, and $\mathrm{c}$ axis, respectively. The symmetry allowed Fermi-type Hamiltonian up to the second power in angular momentum is given as [26]

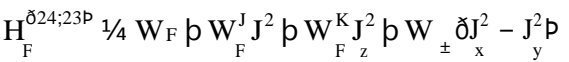

where $\mathrm{W}_{\mathrm{F}}, \mathrm{W}_{\mathrm{F}}^{\mathrm{J}}, \mathrm{W}_{\mathrm{F}}^{\mathrm{K}}$, and $\mathrm{W}_{ \pm}$are the Fermi-type coupling constants. The definition of the K-off-diagonal terms from Eqs. (2) and (3) as implemented in the Pickett's program package [23] has been specified in Ref. [27].

Initially, the quartic and sextic centrifugal distortion constants were fixed to those determined for the ground state and the vibrational energy difference between the $\mathbf{V}_{24_{1}}=1$ and $V_{23}=1$ states was fixed at the infrared value of $10.5 \mathrm{~cm}$. The initial estimation of the Coriolis $G_{c}$ constant was calculated according to the formula [28]

$$
\begin{aligned}
& \mathrm{G}_{\mathrm{C}} 1 / 4 \mathrm{C}_{\mathrm{e}} \mathrm{f}^{\mathrm{c}} \\
& \left.{ }^{24,23}{ }^{1 / 2} \mathrm{x}_{24} \mathrm{x}^{23}{ }^{1=2} \mathrm{P} \quad \mathrm{p} \partial \mathrm{x}^{23}=\mathrm{x}^{24}{ }^{1=2} \mathrm{P}\right]
\end{aligned}
$$

where $\mathrm{f}^{\mathrm{c}}{ }_{24,23}$ is the dimensionless Coriolis constant obtainable from the force field, $x_{24}$ and $x_{23}$ are the harmonic frequencies of the $\mathrm{m}_{24}$ and $\mathrm{m}_{23}$ modes, respectively, and $\mathrm{C}_{\mathrm{e}}$ is the equilibrium rotational constant. The harmonic frequencies were approximated by the IR experimental ones and $\mathrm{C}_{\mathrm{e}}$ by the ground state value. At the MP2/ $6-311++\mathrm{g}(\mathrm{d}, \mathrm{p})$ level of the theory, dimensionless Coriolis constant $f^{c} 24,23=0.14159$ gives according to the Eq. (4) the Coriolis parameter $\mathrm{G}_{\mathrm{c}} \mathrm{rv} 1381 \mathrm{MHz}$. Inclusion of the $\mathrm{G}_{\mathrm{c}}$ constant into the fit and progressive release of the quartic centrifugal distortion constants and 
DE led to its significant improvement with capability to reproduce observed perturbations in low $\mathrm{K}_{\mathrm{a}}$ sequences and to predict higher $\mathrm{J}$ and $\mathrm{K}_{\mathrm{a}}$ transitions for both states. Local $\mathrm{J}$-dependent perturbation in the line sequence of the $V_{23}=1$ state shown in Fig. 4 has been found as a consequence of resonance of $J_{K_{a} K_{a}}{ }^{1 / 4} 190_{19}$ level in $V_{23}=1$ state with $19_{317}$ level in $V_{24}=1$ state with mixing coefficients calculated by SPCAT of $P_{\text {mix }}=0.89$ (see Fig. 5). Impact of this resonance on the observed b-type R-branch transitions is shown in the left part of Fig. 5 where the difference between the frequency $m$ of a given transition in a given excited state and the frequency $\mathrm{m}_{0}$ of the same transition in the ground state scaled by $\left(\mathrm{J}^{00}+1\right)$ value is plot versus $\left(\mathrm{J}^{00}+1\right)$. This plot, obtainable from the AABS package [21], shows a characteristic mirror effect of the $\left(\mathrm{m}-\mathrm{m}_{0}\right)$ quantity resulting from the repulsion of the energy levels being in resonance and was used with a great advantage in many perturbation analyses (see, for example, Refs. [21,24,29]). The maximum perturbation at $\left(\mathrm{J}^{00}+1\right)=20$ corresponds to the shift of the $20_{120} \quad 19_{0} 19$ and $20_{2}$ $18193_{3} 17$ transition lines of more than $800 \mathrm{MHz}$ from the main trend. Subsequent progress in the analysis showed that this perturbation was not so large with respect to several others found for higher $\mathrm{K}_{\mathrm{a}}$ sequences. For example, perturbations at $\left(\mathrm{J}^{00}+1\right)=22$ and $\left(\mathrm{J}^{00}+1\right)=24$, demonstrated in Fig. 5, correspond to the shifts of the involved transition frequencies of more than 3 and $6 \mathrm{GHz}$, respectively, from the visible main trend. It has to be mentioned that those rotational transitions in the $\mathrm{V}_{23}=1$ state having the lower or upper energy level in resonance with corresponding level in $\mathrm{V}_{24}=1$ state, and vice versa, were observed as doublets. Origin of these doublets could be attributed to the internal rotation splitting which is induced by perturbations occurring between both states. Furthermore, in the $\mathrm{V}_{23}=1$ excited state, internal rotation splitting was observed in many R-branch transitions involving the $K_{a}>5$ energy levels and practically all Q-branch series of transitions with $\mathrm{J}^{00}>6$. Internal rotation analysis was not performed in this work and all the transitions showing resolved or partly resolved internal rotation doublets were excluded from the analysis. That is also the reason why the experimental data at the resonant $\mathrm{J}$ values is missing in Fig. 5. As the fitting procedure proceeded, $F_{a b}$ as well as $\mathrm{W}_{ \pm}$constants were also found significant to describe properly the perturbed energy levels. Attempts to fit the experimental data using the Coriolis Hamiltonian with one of $G_{c}$ and $F_{a b}$ fixed to zero were not successful. Both $F_{a b}$ and $G_{c}$ were found as well determined parameters, however, strong correlations between them and $\mathrm{C}$ rotational constants of both states were observed. The experimentally determined $\mathrm{G}_{\mathrm{c}}$ constant was close to the force field value, therefore, to eliminate these correlations, the $\mathrm{G}_{\mathrm{c}}$ constant was in the final fit fixed to the ab initio value. The addition of higher $\mathrm{J}$ and $\mathrm{K}_{\mathrm{a}}$ transitions into the analysis requested to include the centrifugal distortion corrections to the $G_{c}$ and $F_{a b}$ constants which are obtained by the standard expansion of Eq. (2) in $\mathbf{J}^{2}$ and $\mathrm{J}^{2}$. Those producing a significant improvement of the fit were retained. Although the release of the sextic centrifugal distortion constants improved the fit as well, their values revealed quite effective behavior and therefore were kept fixed to the ground state values. Finally, more than 1200 distinct frequency lines involving the transitions up to $\mathrm{J}^{00}=61$ and $\mathrm{K}_{\mathrm{a}}^{00}=10$ and $\mathrm{J}^{00}=59$ and $\mathrm{K}_{\mathrm{a}}^{00}=6$ for the $\mathrm{V}_{24}=1$ and $\mathrm{V}_{23}=1$ state, respectively, were analyzed within the experimental uncertainty. The final set of the spectroscopic constants is presented in Table 1 and the list of the analyzed transitions is given in Table S3 of the electronic supplementary material. It can be seen that the quartic centrifugal distortion constants show a remarkable consistency with those of the ground state in contrast to those determined from the single-state Hamiltonian analysis in Ref. [11]. The experimentally obtained energy difference DE $=10.204536(3) \mathrm{cm}^{-1}$ agrees well with $\mathrm{DE}=10.5 \mathrm{~cm}^{-1}$ obtained from the infrared spectrum.

\section{Conclusions}

High resolution rotational spectra of the cis-methyl vinyl ether have been investigated up to $650 \mathrm{GHz}$. The present work significantly extends the frequency coverage of the cis-methyl vinyl ether rotational spectrum known up to now. More than 4500 distinct frequency lines were assigned which involve the transitions in the vibrational ground state, $\mathrm{V}_{24}=1, \mathrm{~V}_{23}=1$, and $\mathrm{V}_{16}=1$ excited states. To describe correctly numerous local resonances between the levels pertaining to the $V_{24}=1$ and $V_{23}=1$ states, the experimental data were fitted with a model of two vibrational levels interacting by means of the c-type Coriolis and Fermi-type interactions. The resulting sets of the spectroscopic constants reproduce the rotational spectrum of the cis-methyl vinyl ether within the experimental accuracy and allow to search for this molecule in the interstellar medium.

\section{Acknowledgments}

This work has been supported by Ministerio de Ciencia e Innovación (Grants CTQ2010-19008 and Consolider-Ingenio 2010 CSD2009-00038) and Junta de Castilla y León (Grants VA070A08 and VA175U13).

\section{References}

[1] S.B. Charnley, P. Ehrenfreund, Y.J. Kuan, Spectrochim. Acta A 57 (2001) 685704.

[2] R.T. Garrod, E. Herbst, Astron. Astrophys. 457 (2006) 927-936.

[3] R.T. Garrod, S.L.W. Weaver, E. Herbst, Astrophys. J. 682 (2008) 283-302.

[4] S.B. Charnley, M.E. Kress, A.G.G.M. Tielens, T.J. Millar, Astrophys. J. 448 (1995) 232-239.

[5] Z. Peeters, S.D. Rodgers, S.B. Charnley, L. Schriver-Mazzuoli, A. Schriver, J.V. Keane, P. Ehrenfreund, Astron. Astrophys. 445 (2006) 197-204.

[6] G.W. Fuchs, U. Fuchs, T.F. Giesen, F. Wyrowski, Astron. Astrophys. 444 (2005) $521-530$.

[7] Y.-J. Kuan, S.B. Charnley, T.L. Wilson, M. Ohishi, H.-C. Huang, L.E. Snyder, Bull. Am. Astron. Soc. 31 (1999) 942

[8] B.E. Turner, A.J. Apponi, Astrophys. J. 561 (2001) L207-L210.

[9] P. Cahill, L.P. Gold, N.L. Owen, J. Chem. Phys. 48 (1968) 1620-1626.

[10] V.K. Kaushik, Spectrochim. Acta A 34 (1978) 1105-1107.

[11] D. Damiani, E. Gallinella, D.G. Lister, J. Mol. Struct. 95 (1982) 267-270.

[12] M. Fujitake, M. Hayashi, J. Mol. Struct. 127 (1985) 21-33.

[13] R. Meyer, T.K. Ha, M. Oldani, W. Caminati, J. Chem. Phys. 86 (1987) 1848-1857.

[14] B. Cadioli, E. Gallinella, U. Pincelli, J. Mol. Struct. 78 (1982) 215-228.

[15] I.S. Ignatyev, M. Montejo, T. Sundius, F. Partal Ureña, J.J. López González, Chem. Phys. 333 (2007) 148-156.

[16] D.A. McWhorter, S.B. Cupp, C.Y. Lee, B.H. Pate, J. Mol. Spectrosc. 193 (1999) $150-158$.

[17] A.M. Daly, C. Bermúdez, A. López, B. Tercero, J.C. Pearson, N. Marcelino, J.L. Alonso, J. Cernicharo, Astrophys. J. 768 (2013) 81.

[18] A. López, B. Tercero, Z. Kisiel, A.M. Daly, C. Bermúdez, H. Calcutt, N. Marcelino, S. Viti, B.J. Drouin, I.R. Medvedev, C.F. Neese, L. Pszczolkowski, J.L. Alonso, J. Cernicharo, Astron. Astrophys., 2014. http://dx.doi.org/10.1051/0004-6361/ 201423622.

[19] L. Kolesniková, A.M. Daly, J.L. Alonso, B. Tercero, J. Cernicharo, J. Mol. Spectrosc. 289 (2013) 13-20.

[20] L. Kolesniková, B. Tercero, J. Cernicharo, J.L. Alonso, A.M. Daly, B.P. Gordon, S.T Shipman, Astrophys. J. 784 (2014) L7.

[21] Z. Kisiel, L. Pszczółkowski, I.R. Medvedev, M. Winnewisser, F.C. De Lucia, E. Herbst, J. Mol. Spectrosc. 233 (2005) 231-243. 
[22] J.K.G. Watson, in: J.R. Durig (Ed.), Vibrational Spectra and Structure, vol. 6, Elsevier, Amsterdam, 1977, pp. 1-89.

[23] H.M. Pickett, J. Mol. Spectrosc. 148 (1991) 371-377.

[24] Z. Kisiel, L. Pszczółkowski, B.J. Drouin, C.S. Brauer, S. Yu, J.C. Pearson, I.R. Medvedev, S. Fortman, C. Neese, J. Mol. Spectrosc. 280 (2012) 134 144.

[25] P.R. Bunker, P. Jensen, Molecular Symmetry and Spectroscopy, NRC Research Press, Ottawa, 2006.
[26] V.I. Perevalov, V.G. Tyuterev, J. Mol. Spectrosc. 96 (1982) 56-76.

[27] R.A.H. Butler, D.T. Petkie, P. Helminger, F.C. De Lucia, J. Mol. Spectrosc. 220 (2003) 150-152

[28] D. Papousek, M.R. Aliev, Molecular Vibrational-Rotational Spectra, Academia, Prague, 1982.

[29] Z. Kisiel, E.B. Jaworska, R.A.H. Butler, D.T. Petkie, P. Helminger, I.R. Medvedev, F.C. De Lucia, J. Mol. Spectrosc. 254 (2009) 78-86. 